\title{
DESIGN AND IMPLEMENTATION OF SIMPLE ELECTRON DETECTORS FOR ACCELERATOR DIAGNOSTICS*
}

\author{
R.A. Rosenberg ${ }^{\dagger}$ and K.C. Harkay, Advanced Photon Source \\ Argonne National Laboratory, Argonne, IL 60565, USA
}

\begin{abstract}
We have constructed a compact, planar retarding field analyzer (RFA) for the diagnostics of low-energy, background electrons in a high-energy particle accelerator. Bench measurements of the analyzer have been made to characterize it, and the results are reasonable in light of models of this type of analyzer. We have recently implemented an upgraded detector, based on a "Bessel Box" design. This detector has the advantage of superior energy dispersion, but has lower signal levels. Here we present bench measurements and initial data from the Advanced Photon Source.
\end{abstract}

\section{INTRODUCTION}

Electrons in accelerators are ubiquitous. They can be produced directly by irradiation of vacuum chamber surfaces by x-rays, ions, and other particles, or indirectly by bombardment of electrons, which leads to production of secondary electrons. Under most circumstances, electrons are not detrimental to accelerator performance; however, there are operating conditions that can lead to amplification of the electrons. If the number density of the electrons becomes sufficiently large, the electrons can lead to degradation of the particle beam, either by direct interaction or through electron-stimulated desorption of gases. The dense pockets of electrons are termed an electron cloud (EC).

In order to directly measure the properties of the EC, a special vacuum chamber was installed in the Advanced Photon Source (APS) storage ring. The chamber contains 10 rudimentary, retarding-field electron energy analyzers [1], which were used to examine the intensity and electron energy distribution of the electrons in the storage ring under different operating conditions. Similar RFAs were also employed at the Proton Storage Ring at Los Alamos National Laboratory [2] and the Beijing Electron Positron Collider ring at the Institute of High Energy Physics [3] to diagnose the electrons produced at these facilities.

Although the RFA was successful at providing qualitative electron energy analysis, it could only obtain spectra in an integrated form. In order to overcome this limitation, an energy-dispersive analyzer was developed based on the "Bessel Box" design [4,5]. In this paper, the design and characterization of both types of analyzers will be described.

\footnotetext{
*This work was performed at the Advanced Photon Source and supported by the U.S. Department of Energy, Office of Basic Energy Sciences under Contract No. W-31-109-ENG-38.

†rar@aps.anl.gov
}

\section{DESIGN}

\subsection{Retarding-Field Analyzer}

The RFA has been described previously [1], so only a brief description will be given here. In its simplest configuration, the instrument consists of a retarding grid followed by a collector. Spherically symmetric grids should be used if possible, but geometric constraints forced us to opt for planar grids. The theoretical transmission of the RFA is shown in Fig. 1.
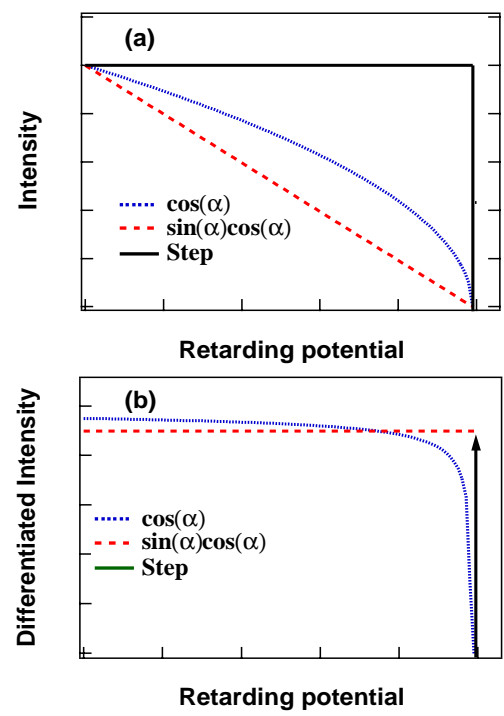

Figure 1: (a) Theoretical transmission of a planar RFA. Solid line - ideal case for a parallel, nondivergent monoenergetic beam of energy $\mathrm{U}_{0}$. Dashed line transmission assuming the electrons originate from a point source from a parallel surface with an angular distribution; $\mathrm{P}(\alpha) \mathrm{d} \alpha=2 \sin \alpha \cos \alpha \mathrm{d} \alpha$, where $\alpha$ is the angle between the electrons and the surface normal [6]. Also shown is the transmission curve for a $\cos \alpha$ distribution (dotted line). (b) Differentiated signal for the three cases.

The RFA consists of two 70 lines/inch (90\% transmission) copper grids and a collector. The first grid is grounded to present a uniform field to the incoming electrons. The second grid is biased at a retarding potential $\left(\mathrm{E}_{\mathrm{r}}\right)$ such that only electrons with kinetic energies greater than $\mathrm{E}_{\mathrm{r}}$ are transmitted to the collector. The collector is graphite-coated to lower the secondary electron yield (SEY), and biased at $45 \mathrm{~V}$ with a battery to increase the collection efficiency. The assembled detector was mounted behind a slotted vacuum 
penetration on a 2.75-inch Conflat flange with two feedthoughs that were used to provide the retarding voltage and measure the collector current.

\subsection{Bessel Box Analyzer (BBA)}

The BBA, sometimes referred to as a "pill box," is perhaps the simplest energy-dispersive analyzer. It is shown schematically in Fig. 2. Basically, it consists of entrance and exit apertures held at potential $V_{a}$, the body with potential $\mathrm{V}_{b}$, and a collector. At the center of the body is a circular "stop," whose diameter is slightly larger than the entrance aperture, which prevents direct, on-axis electrons from being transmitted through the exit aperture. The stop is also maintained at $\mathrm{V}_{\mathrm{b}}$. The difference between $\mathrm{V}_{\mathrm{b}}$ and $\mathrm{V}_{\mathrm{a}}, \Delta \mathrm{V}$, determines the pass energy of the analyzer. Electrons with too low an energy will be repelled by $V_{b}$, while electrons with too high an energy will not be deflected enough to be transmitted through the exit aperture and collected (yielding the collector current, $i_{c}$ ). The performance and characteristics of this type of analyzer have been described previously [4,5].

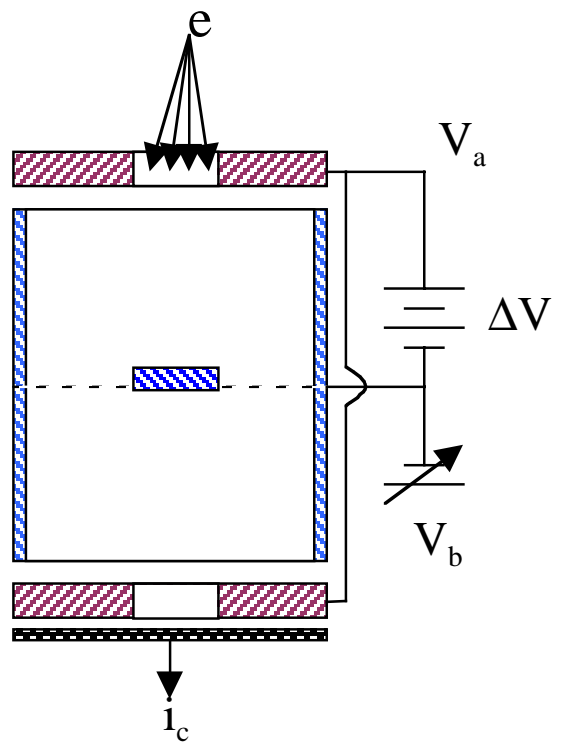

Figure 2: Schematic diagram of the Bessel Box analyzer. (Symbols are described in text.)

The BBA depicted in Fig. 2 was designed to mount on a standard 2.75-inch flange so that it could be used in place of the RFAs used previously. In addition to the apertures, body, and collector, it also had an electrically isolated front grid and cylindrical shield that were maintained at ground potential. These served to block stray electrons from entering the analyzer and to shield the electrons from the electron gun used for testing from the fields produced by the analyzer. The collector was a plate coated with graphite and biased with a battery at $30 \mathrm{~V}$. All parts were machined from aluminum and coated with graphite to reduce the SEY.

\section{PERFORMANCE}

\subsection{RFA Laboratory Measurements}

All RFAs were calibrated on the bench using an $\sim 1-\mu \mathrm{A}$ electron beam from a low-energy electron gun. The gun was mounted directly opposite the detector so the electron beam was parallel to the axis of the detector and approximately nondivergent. Fig. 3(a) shows the detector response and the derivative for a typical detector. The average resolution for all detectors is $\sim 4 \%(\Delta \mathrm{E} / \mathrm{E})$. Due to the idealized nature of the beam from the electron gun, the detector response approximates the step function shown in Fig. 1(a), and the derivative approaches the delta function shown in Fig. 1(b). At retarding voltages greater than zero, the retarding grid efficiently collects and focuses the scattered electrons onto the collector. This accounts for the increased signal at positive energies. However, as the voltage on the retarding grid approaches that of the battery $(45 \mathrm{~V})$, electrons are deflected away from the collector, thereby decreasing the signal.
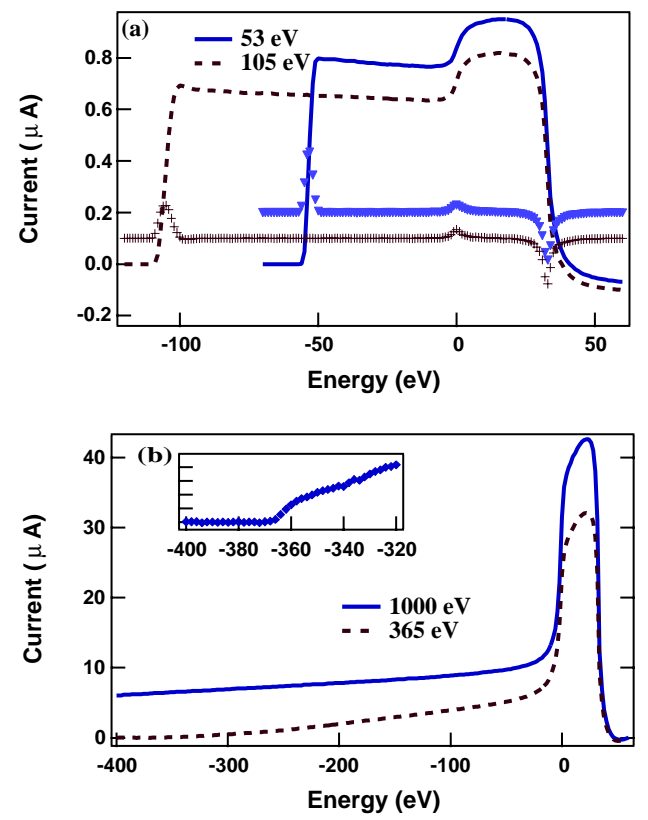

Figure 3: (a) Transmission curves for a monoenergetic electron beams directed along the axis of the analyzer for energies of 53 and $105 \mathrm{eV}$. The differentiated signal is also shown. (b) Transmission curves of monoenergetic electrons $(365,1,000 \mathrm{eV})$ scattered from an aluminum target. The inset shows the differentiated signal of the $365-\mathrm{eV}$ beam near the transmission threshold.

For insight into the detector behavior under more realistic conditions, bench measurements were also taken of electrons scattered from a surface. In this case, a monoenergetic beam of electrons was directed at an aluminum target at an angle of $\sim 30$ degrees to the surface normal. The analyzer was positioned to detect electrons scattered at $\sim 30^{\circ}$. Representative data are shown in Fig. 3(b) for $1,000-\mathrm{eV}$ and $365-\mathrm{eV}$ electrons. Except near zero $\mathrm{V}$, the data monotonically increase in a fashion 
reminiscent of the theoretical curves represented by the dashed or dotted lines in Fig. 1(a). It is important to note that the electrons scatter from the surface elastically and inelastically, so the data shown in Fig. 3(b) contain a large contribution from low-energy electrons. The inset in Fig. 3 (b) shows the differentiated signal resulting from scattering of the $365-\mathrm{eV}$ electrons in the vicinity of the elastically scattered beam. Although there is considerable broadening, these data show a step-like increase in the signal in a fashion similar to the dashed or dotted lines in Fig. 1(b).

\subsection{BBA Laboratory Measurements}

Figure 4 shows data taken with the BBA using $340-\mathrm{eV}$ electrons scattered from an aluminum surface approximately $2 \mathrm{~cm}$ from the entrance to the analyzer. The large peak at low energies is due to secondary electrons scattered from the target surface. The inset shows details of the elastically scattered primary peak, which indicates a resolution of about $1.4 \mathrm{eV}$. The data were acquired using a $30-\mathrm{V}$ battery to supply $\Delta \mathrm{V}$; another $30-\mathrm{V}$ battery was used to bias the collector to increase its efficiency.

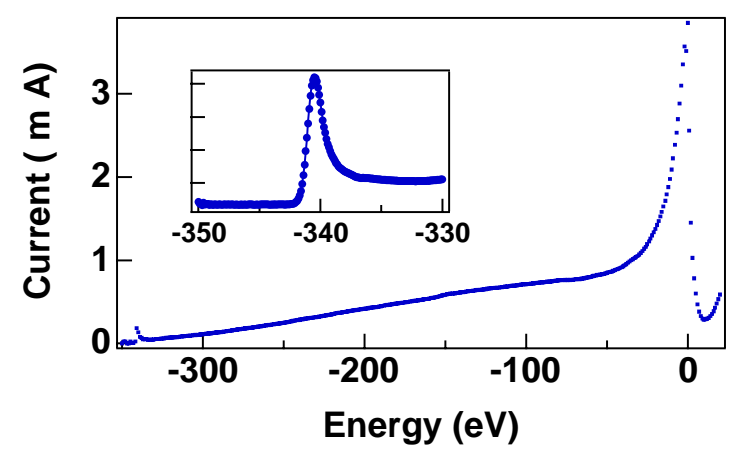

Figure 4: Spectrum of 340-eV electrons scattered from an aluminum surface using the BBA. The inset shows a detailed scan of the elastically scattered electrons.

\subsection{Accelerator Measurements}

Figure 5 shows measurements taken with both types of analyzers at two locations under the same conditions (20 bunches spaced by eleven 2.8 -ns rf buckets with $2 \mathrm{~mA}$ per bunch). Figure 5(a) shows data taken with detectors located $0.2 \mathrm{~m}$ upstream from the main source of electrons (the photon absorber), while Fig. 5(b) shows results from detectors $2 \mathrm{~m}$ upstream. The signal from the detectors at $0.2 \mathrm{~m}$ is dominated by the electrons produced by photoemission from the absorber, whereas the spectra from detectors at $2 \mathrm{~m}$ are expected to show significant contributions from electrons produced by beam-induced multipacting [7]. The BBAs show considerably more detail than the RFAs, and the low-energy cutoff seen in the BBA spectrum in (b) cannot be easily explained. It is interesting to note that this cutoff energy is a function of both bunch spacing and current. The maximum signal from the BBA is about 500 times less than that from the
RFA in (b) and about 25 times less than in (a). Analysis of these data is ongoing.
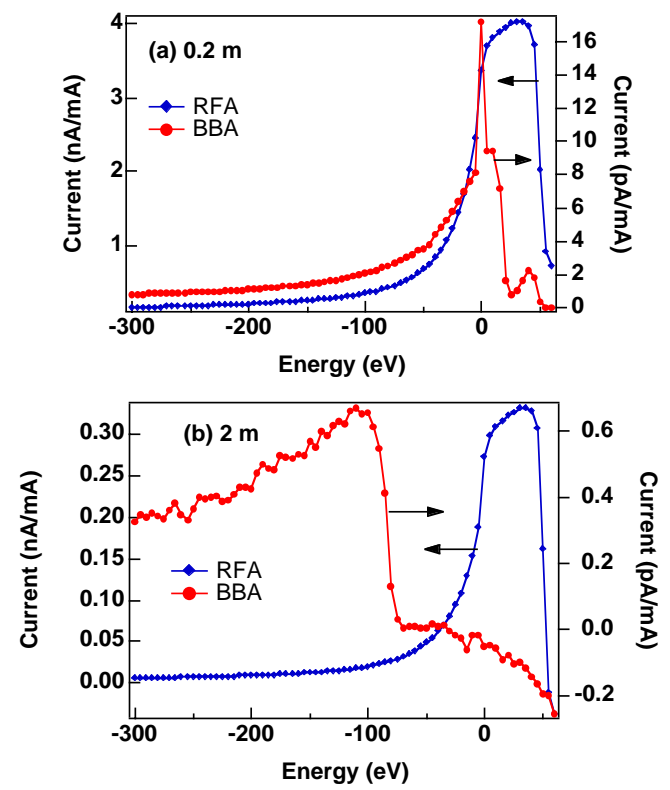

Figure 5: Electron energy spectra from RFAs and BBAs installed in the APS storage ring located $0.2 \mathrm{~m}$ (a) and $2 \mathrm{~m}$ (b) upstream from a photon absorber.

\section{CONCLUSIONS}

The design and performance of two simple electron energy analyzers for accelerator diagnostics is described. The RFA is simple to construct and efficient, but analysis of the energy spectra is complicated. While the BBA has relatively poor transmission, it offers the potential of direct analysis of the energy spectrum. Preliminary results from the BBA installed in the APS storage ring are intriguing. In the future, we will devote more time to experiments and analysis using the BBA. We also hope to incorporate an electron multiplier for signal amplification, which should allow us to conduct time-resolved measurements.

\section{REFERENCES}

[1] R.A. Rosenberg and K.C. Harkay, Nucl. Instr. Meth. [A] 453, 507 (2000).

[2] A. Bowman, Proceedings of ICFA 8th Advanced Beam Dynamics Mini-Workshop on Two-Stream Instabilities, Feb 16-18, 2000, Santa Fe, NM, http://www.aps.anl.gov/conferences/icfa/proceedings.h tml.

[3] Z. Guo, et al, this conference.

[4] I. Lindau, J.C. Helmer, and J. Uebbing, Rev. Sci. Instr. 44, 265 (1973).

[5] J.H. Craig, Jr., and W.G. Durrer, J. Vac. Sci. Technol. A 7, 3337 (1989).

[6] T.H. DiStefano and D.T. Pierce, Rev. Sci. Instr. 32, 180 (1970).

[7] K.C. Harkay and R.A. Rosenberg, Proceedings of the 1999 Particle Accelerator Conference, p. 1641 (1999). 